\title{
Correction: Transient acute-onset tetraparesis in a COVID-19 patient
}

\section{Andrea Giorgianni - Gabriele Vinacci - Edoardo Agosti (1) - Lucia Princiotta Cariddi - Marco Mauri • Fabio Baruzzi • Maurizio Versino}

Published online: 27 July 2020

(c) International Spinal Cord Society 2020

\section{Correction to: Spinal Cord \\ https://doi.org/10.1038/s41393-020-0493-8 \\ published online 2 June 2020}

In the original version of this article, one affiliation was inadvertently omitted. The affiliation "Clinical and
Experimental Medicine and Medical Humanities, Center of Research in Medical Pharmacology, University of Insubria, Varese, Italy" has now been added to the article in association with the author Lucia Princiotta Cariddi.

This has been corrected in both the PDF and HTML versions of the Article. 